\title{
Homozygosity mapping of the gene for Hallervoden-Spatz syndrome to chromosome 20p12.3-p13
}

Todd Taylor, Michael Litt, Patricia Kramer, Massimo Pandolfo, Lucia Angelini, Nardo Nardocci, M.Pineda, Suzanne Davies, Haruo Hattori, Peter J. Flett, M. Roberta Cilio, Enrico Bertini \& Susan J. Hayflick

Nature Genetics 14, 479-481 (1996).

In Fig. 1 the $\mathrm{Hs} 9$ pedigree was inadvertently included. The other pedigrees, the figure legened and the text are correct. Family HS9 provides preliminary evidence for locus heterogeneity in Hallervorden-Spatz syndrome. These completed data will be presented in a forthcoming manuscript.

Yeast SAS silencing genes and human genes associated with AML and HIV-1 Tat interactions are homologous with acetyltransferases

Cherly Reifnyder, Joanna Lowell, Astrid Clarke \& Lorraine Pillus

Nature Genetics 14, 42-49 (1996).

Owing to a printing error, the lightly shaded residues in Figure 3 appeared a little too light. The correct version of the figure is shown below.

\begin{tabular}{|c|c|}
\hline 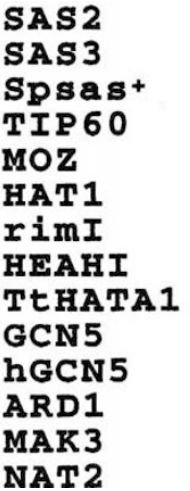 & 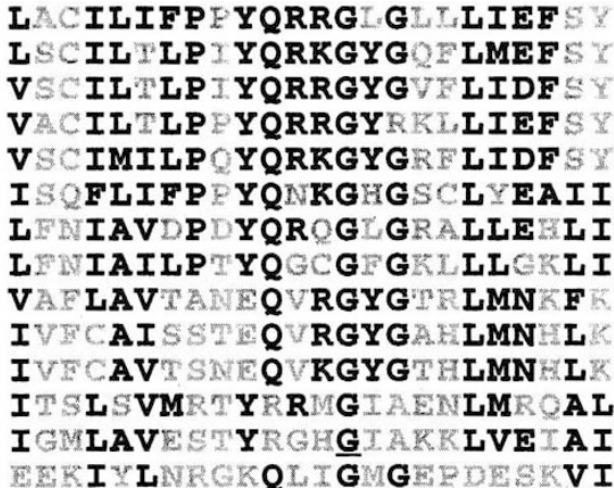 \\
\hline
\end{tabular}

